\title{
HEIDEGGER Y BOLAÑO: FUNCIÓN, GESTO Y ÁNIMO EN EL LENGUAJE
}

Heidegger and Bolaño: Function, gesture and mood in the lenguage

\author{
DANIEL MICHELOW BRIONES \\ Universidad Alberto Hurtado (Chile) \\ daniel.michelow@gmail.com
}

Resumen

$\mathrm{Al}$ momento de comparar dos obras disímiles en términos de lo que aquí llamaremos el gesto lingüístico, pero similares en cuanto a su comprensión fundamental del decir respecto de su función, se vuelve clara la necesidad de expandir la metódica de acceso a tal fenómeno e incluir el momento analítico clave del ánimo del lenguaje. El presente análisis tiene como meta lograr tal expansión, desde el punto de partida ontológico que se propone en Ser y tiempo, para llevarlo hasta un puerto - en apariencia- tan disímil como es la obra literaria de Roberto Bolaño.

Palabras clave: Heidegger; Bolaño; lenguaje; literatura; ontología.

Abstract

When comparing two works, that are dissimilar in terms of what we will call here the linguistic gesture, but similar in terms of their fundamental understanding of the concept with respect to its function, the need to expand the access to such phenomena becomes clear, and to also include the key analytical moment of the mood of the language. The purpose of this analysis is to achieve expansion, from the proposed ontological starting point in Being and time, to take it to a port -apparently-as different as the literary work of Roberto Bolaño.

Key words: Heidegger; Bolaño; language; literature; ontology.

\section{FUNCIÓN}

El presente ensayo tiene como objetivo general dar cuenta del aspecto de la metódica heideggeriana relativa a la descripción y acceso al fenómeno del lenguaje. Específicamente a la determinación que se le otorga en Ser y tiempo (en adelante ST) como "decir" (Rede). Someramente se abordará también su evolución tras el giro (Kehre) aspirado por el pensamiento de Heidegger. En términos específicos, se llevará a cabo este análisis en relación comparativa al uso y comprensión que adquiere el lenguaje en el contexto literario y poético de la obra de Roberto Bolaño.

Debido al tipo de objetivos propuestos es urgente adelantarse a las interrogantes naturales que se podrán originar respecto de un planteamiento aparentemente poco homogéneo como el presente. La primera interrogante guarda relación con la necesidad de llevar a cabo una comparación que sobrepasa la obra misma de Heidegger para revelar la metódica de este. La segunda interrogante es aquella respecto de la obra de 
Bolaño como representante adecuado de lo literario, en vistas a las delimitaciones y exigencias heideggerianas para un análisis de este tipo.

Respecto de la primera interrogante se debe aclarar que aquello que se trata bajo el termino "metódica" no es un procedimiento que se le imponga de antemano al objeto analizado, sino que más bien es un modo del trato, que surge desde el ocuparse mismo con tal objeto. La metódica heideggeriana no constituye un método en el sentido tradicional que efectúe su modo particular de acceso a los objetos analizados de modo puramente formal, sino que requiere ser probada concretamente. Es justamente por esto que el propio Heidegger elige a menudo como procedimiento filosófico la exégesis de otras obras, pues es solo en el "choque" de proyectos donde se revela la arquitectura interna de los fenómenos y es, de igual manera, la razón por la que el presente análisis tiene lugar bajo la forma de una relación comparativa.

Para responder la segunda interrogante es necesario previamente aclarar aquello que Heidegger comprende como literatura y su relación con el pensamiento filosófico. Encontramos indicios en su célebre Carta sobre el humanismo en que los destinos de la poesía y del pensar son definitivamente entrelazados:

El lenguaje es la casa del ser. En su morada habita el hombre. Los pensadores y poetas son los guardianes de esa morada. Su guarda consiste en llevar a cabo la manifestación del ser, en la medida en que, mediante su decir, ellos la llevan al lenguaje y allí la custodian (Heidegger GA 9, 1).

Pensar y poetizar son -a partir de esta descripción-modalidades de lo mismo, a saber de la guarda decidora de la manifestación o apertura del ser. Sin ir más lejos, adquiere el contexto literario de análisis del lenguaje relevancia en un nivel ontológico porque en él tiene el habla un tipo de concreción propia. En cuanto a Bolaño como representante de lo literario y poético en el sentido del "llevar a cabo la manifestación del ser" es nuevamente posible recurrir a lo dicho por Heidegger, en este caso respecto de Hölderlin:

Hölderlin no se ha escogido porque su obra, como una entre otras, realice la esencia general de la poesía, sino únicamente porque está cargada con la determinación poética de poetizar la propia esencia de la poesía. Hölderlin es para nosotros en sentido extraordinario el poeta del poeta (Heidegger GA 4, 1).

El requisito aplicado por Heidegger para elegir a Hölderlin como el poeta representante de lo poético por sobre otros nombres insignes es la presencia de una metaliteratura que atraviesa su obra, una poesía autorreferente en la que por tanto y según lo descrito es tematizado de modo poético, el modo en que la poesía tematiza, esto es acredita, la manifestación del ser. ¿Cabe Bolaño en esta categoría metaliteraria?

\footnotetext{
${ }^{1}$ Las citas de Heidegger dentro del texto señalarán la numeración de las obras completas (GA). La paginación corresponderá por otra parte a la traducción castellana elegida en cada caso. La edición castellana utilizada estará citada al final del escrito en "Obras citadas".
} 
¿Es el autor chileno un novelista de la novela? ¿Y por sobre todo, hay en Bolaño algo así como una tematización del ser? Respecto de la metaliteratura se puede responder sin rodeos en forma afirmativa, pues es probable que no haya otro autor contemporáneo que haya dedicado su obra tan seguro de sí a dar cuenta de la existencia literaria y, por medio de esta, de la tarea fundamental y función de la literatura. En Bolaño se desarrolla como nunca antes una literatura que construye un relato en el que la literatura misma es el telón de fondo contra el que los personajes se desarrollan y justamente por ello son siempre aquellos dedicados a la literatura -críticos, escritores, lectores empedernidos, académicos-aquellos que presienten la trama de los acontecimientos en los que se ven inadvertidamente envueltos. A la pregunta respecto de la presencia de una acreditación patente del ser en su obra nos abocaremos más adelante. Así parece la obra de Bolaño cumplir los primeros requisitos.

Pero más allá del contexto técnico que hemos descrito para la posibilidad de una comparación entre ambos, es necesario decir - esto tiene que ver con el modo en que entenderemos aquí la literatura- que es posible confrontar ambas obras porque de cierto modo el ejercicio literario, del que Bolaño es un representante, no se aboca solo al relato de hechos -sea desde el genre que sea- de antemano dados en el mundo, sino que dar cuenta por medio de la interpretaciòn del mundo que habitamos y cómo lo habitamos.

Ahora bien, respecto de lo que aquí llamamos el triple acceso al fenómeno del lenguaje en la obra de Heidegger, específicamente en ST, se podrá encontrar el primer indicio en el capítulo segundo de la introducción, específicamente en el $\S 7$, donde se entrega una descripción corta del fenómeno de "decir" bajo el título El concepto de logos. Ahí Heidegger nos indica que en primer lugar para recuperar el sentido del lenguaje que él pretende implementar, esto es para dar paso a su significado esencial, será necesario apartarse de las variadas interpretaciones que ha dado la filosofía al logos griego, así como "juicio", "concepto", "valor" y "razón", pues estas contienen necesariamente un momento de "toma de posición" (p. 42) respecto de lo mentado. Este significado esencial se encuentra por primera vez expresado de manera decidida en Aristóteles que determina la función del logos como un $\alpha \pi \circ \phi \alpha$ liv\& $\sigma \theta \alpha$, esto es hacer ver, hacer patente para el otro lo que se habla (p. 42). Es precisamente este sentido del logos el que Heidegger quiere traducir e interpretar con la sencilla palabra "decir". Cuando el decir es un decir genuino, descubre lo dicho, no lo pone en relación con otra cosa, no es "relacional" (p. 43), sino que descubridor, lleva a cabo la simple función de dejar ver lo mentado en sí mismo. El decir filosófico así como el literario se instauran como el lugar (la casa) para la manifestación del ser, esto quiere decir, el ámbito en el que el despliegue histórico como tal de la totalidad del ente se hace patente. A tal despliegue, como es sabido, le llamará Heidegger en su filosofía tardía el "acontecimiento apropiador" (Ereignis).

A partir de esta descripción del fenómeno del decir en ST es posible construir un puente con la concepción de Bolaño de la función de la literatura. Acerca de esta función es posible encontrar variadas referencias a lo largo de sus principales escritos, 
que componen el corpus de su obra como Los detectives salvajes, El gaucho insufrible, 2666 o El tercer Reich. Este análisis tomará en cuenta principalmente 2666 y Una Novelita Lumpen, pues en estas parecen darse las condiciones adecuadas para la comparación planeada. Un buen punto de partida -señalado por Ignacio Echevarría en la introducción a la primera edición de 2666- es la referencia del propio Bolaño en notas privadas a un "centro oculto" de su obra. Un centro oculto latente tras el "centro físico" en el que se desenvuelven los personajes comúnmente (p. 480). El mismo Echevarría aventura una interpretación acerca de este centro oculto:

Hay razones para pensar que ese centro físico sería la ciudad de Santa Teresa, fiel trasunto de Ciudad Juárez, en la frontera de México con Estados Unidos. Allí convergen, al cabo, las cinco partes de la novela; allí tienen lugar los crímenes que configuran su impresionante telón de fondo (y de los que, en un pasaje de la novela, dice un personaje que "en ellos se esconde el secreto del mundo"). En cuanto al "centro oculto"..., ¿no lo estaría indicando precisamente esa fecha, 2666, que ampara la novela entera? (Echevarría, 2004, p. 480)

El centro físico es lo que está a la vista y es evidente, que no requiere ser develado, aquello a lo que Heidegger llamó en su obra la cotidianeidad (Alltäglichkeit). El centro oculto por otro lado es lo que aun soterrado ejerce su fuerza aunadora y apropiadora. Reúne en el caso de Bolaño el relato y las cientos de voces dispares que pueblan su obra y se apropia de los destinos de estas. Todos sus personajes, de algún u otro modo, son llevados a ese centro, atraídos por su poder, tanto si están conscientes de ello o no. La referencia a lo oculto, a lo que se retrae -como el ser en Heidegger-no es exclusiva de 2666. Otra referencia a esta fuerza (además de "el secreto del mundo" recién mencionado) puede ser encontrada en Una novelita lumpen. Ahí el personaje principal nos dice en las últimas páginas de la obra:

y me ponía a pensar en el significado de la frase "cambiar nuestra suerte", una frase que para mí no tenía ningún significado, por más vueltas que le diera, porque la suerte no se puede cambiar, o existe o no existe, y si existe no hay manera de cambiarla, y si no existe somos como pájaros en una tormenta de arena, solo que no nos damos cuenta, por supuesto, tal como dice la canción de Luciano Marchetti: "somos pájaros en la tormenta, nadie lo experimenta". Aunque yo creo que hay gente, gente muy desdichada o con muy mala suerte, que sí se da cuenta de ello (Bolaño, 2009, p. 13).

Y ya directamente al final de esta novela corta:

Durante muchos días, sin embargo, estuve a la espera de una mala noticia. Leía la prensa (no todos los días porque no teníamos dinero para comprar el periódico a diario), veía la tele, escuchaba las noticias de la radio en la peluquería, temerosa de encontrar la figura final de Maciste tirado en el suelo, en medio de un charco de sangre (su sangre fría), y junto a él las fotos tipo carnet del boloñés y del libio, mirándome con nostalgia desde una página o desde la pantalla de nuestra tele que ya era realmente nuestra y no de nuestros padres muertos, como si las fotos de 
ellos, los asesinos y la víctima, el asesino y las víctimas, fueran la señal de que en el exterior aún persistía la tormenta, una tormenta que no estaba localizada sobre el cielo de Roma, sino en la noche de Europa o en el espacio que media entre planeta y planeta, una tormenta sin ruido y sin ojos que venía de otro mundo, un mundo que ni los satélites que giran alrededor de la Tierra pueden captar, $\mathrm{y}$ donde existía un hueco que era mi hueco, una sombra que era mi sombra (p. 39).

De este modo es posible sacar la conclusión, como primer paso para la presente exposición, que tanto Heidegger como Bolaño se mueven dentro de una comprensión unívoca de la función del lenguaje como descubridor de lo que retrotrayéndose reúne y atrae, esto quiere decir se apropia de los destinos humanos.

\section{GESTO}

Como se ha dicho en la primera parte de este ensayo el dejar ver es la función del decir. Estructuralmente diferente es el modo en que tal función es implementada, por eso no es casualidad que en ST, dentro del mismo capítulo donde se encuentra el decir pensado como existenciario, se hable a su vez, bajo el título "La tarea de una destrucción de la historia de la ontología", de lo que aquí será llamado el gesto. El gesto de despliegue de la función descubridora del decir es en la obra temprana de Heidegger la destrucción. ST está fundamentalmente determinado por el gesto destructor en el ámbito del cumplimiento de las posibilidades del Dasein. Respecto de la tarea planteada nos dice el propio Heidegger:

Si se quiere que la pregunta misma por el ser se haga transparente en su propia historia, será necesario alcanzar una fluidez de la tradición endurecida, y deshacerse de los encubrimientos producidos por ella. Esta tarea es lo que comprendemos como la destrucción, hecha al hilo de la pregunta por el ser, del contenido tradicional de la ontología antigua, en busca de las experiencias originarias en las que se alcanzaron las primeras determinaciones del ser, que serían en adelante las decisivas (Heidegger GA 2, 32).

El gesto destructor del revelar se debe por tanto presentar en primera instancia como destrucción de la historia, esto es de las posibilidades heredadas por el Dasein de ser su existencia. Destruir la historia es por tanto combatir la falta de fluidez y transparencia en la que han caído las determinaciones lingüísticas que recogen nuestra experiencia del ser. El plan de ST es la destrucción de esas determinaciones heredadas, mostrándolas, en la mayoría de los casos, como meras derivaciones para las que su propio origen ha quedado oculto.

Para Heidegger el sentido del ser está oculto en primer término tras la constelación conceptual de la que se ha servido la tradición filosófica y urge la implementación de un plan radical de acción, pues el presentimiento que parece mover toda la obra en cuestión es la de un ocultamiento y anquilosamiento progresivo de los 
modos históricos de darse del ser con el riesgo además de que tal estado se vuelva irremediable.

Lo descrito explica en gran parte una cierta violencia conceptual en el modo de expresión, tan célebre como criticado, que ha utilizado Heidegger para llevar a cabo su proyecto filosófico. Un ejemplo de esta "lucha" por devolverle fluidez al lenguaje sucede en una palabra que se ha utilizado - sin ir más lejos- en el presente ensayo. Se trata del concepto de "existenciarios" (Existenzialien), que vendría a representar el equivalente fundamental-ontológico de las "categorías" metafisicas surgidas en Aristóteles. Pero esto no es una simple variación en el modo en que nos referimos a una cosa dada, sino que más bien implica una transformación tanto del lenguaje mismo como de lo mentado en la palabra. Esta transformación ocurre en ST solo de manera destructiva, es decir, quitándole toda posibilidad de subsistencia - más que como un residuo histórico- a la palabra transpuesta. Esta destrucción no es un movimiento negativo que tenga como finalidad descartar y desechar lo que traspasa y hereda la tradición, sino que proponerlo en el contexto de nuevas perspectivas -posibilidades de ser, diría Heidegger-. La filosofía y la literatura son actividades limítrofes en el sentido de que están precisamente encargadas de decidir el trazado del límite de la experiencia posible.

No hay duda de que Bolaño está al igual que Heidegger al tanto de las dificultades que conlleva el concretar en el lenguaje la manifestación del ser, o parafraseando dicha aseveración en los términos del autor chileno, llevar a la palabra poética el centro oculto. Por esto es posible encontrar en la obra de Bolaño variadas referencias al escritor como un guerrero "que está siempre luchando" y que sabe "que al final, haga lo que haga, será siempre derrotado" (Bolaño, 2004, p. 323). O en otras ocasiones, esta vez haciendo referencia a ese centro oculto directamente: "La literatura es una máquina acorazada. No se preocupa de los escritores. A veces ni siquiera se da cuenta de que estos están vivos. Su enemigo es otro, mucho más grande, mucho más poderoso, y que a la postre la terminará venciendo" (Bolaño, 2013, p. 150).

Pero a pesar de que Bolaño entiende al escritor como un guerrero, esto es a la literatura -así como Heidegger al pensar- como un esfuerzo al borde de sus posibilidades y compartiendo ambas obras en lo fundamental esa función del lenguaje, difieren radicalmente en el gesto de implementación de tal función. El gesto heideggeriano determinado por la destrucción de la historia de la ontología es violento y urgente. Heidegger recupera formas y significados arcaicos en desuso, pero también inventa y crea nuevos términos y signos. Acopla o divide inusualmente. Distorsiona, modifica, verbaliza y sustantiva de modo inesperado ${ }^{2}$. Se hace de todas las herramientas

\footnotetext{
${ }^{2}$ Los ejemplos son incontables, podemos mencionar muchos de los conceptos fundamentales de ST como "ser-en-el-mundo" (In-der-Welt-sein), en el que se une un conjunto de palabras para su sustantivación, "estado de descubierto" (Entdecktheit) una sustantivación neológica, ser-ahí (Da-sein) en el que se divide la palabra original para acentuar su significado originario o el ya mencionado existenciario (Existenzialien), todos neologismos con estructuras lingüísticas diferentes, pero siempre con el mismo sentido: abrir un espacio no-metafísico de expresión para el ser. La radicalidad de esta búsqueda se hace patente en que incluso la palabra "ser", a lo largo de su obra, sufre diversas transformaciones por ejemplo el cambio de "Sein" a "Seyn" 
a mano para lograr que el logos anquilosado fluya nuevamente. Pero nunca de modo unilateral, sino que siempre desde la exigencia ontológica de aquello que requiere un lugar propio en el decir. El gesto tiene su medida en la necesidad que emana desde la función del decir. Es por esto que la crítica superficial al gesto lingüístico en Heidegger motejándolo como una simple jerga al estilo de Adorno (2005), en su famosa obra "Jerga de la autenticidad", parece no hacerle mella, principalmente porque esta crítica no conoce la relación y dinámica entre función y gesto. No se pretende aquí en ningún caso aseverar que el gesto lingüístico en Heidegger es inmune a críticas, más bien que para sopesarlo en su justa medida es necesario comprenderlo primeramente como una herramienta a disposición de la función descubridora del decir filosófico. Esto es justamente lo que Adorno no comprende, pues para él el lenguaje es una mera herramienta comunicativa. Para el tipo de análisis al que no se le ha vuelto cuestión el despliegue histórico y la transformación del aparecer del ente en su totalidad desde el aparecer mismo, todo decir del ser suena a jerga.

El lenguaje descubridor, para justamente descubrir lo oculto, deberá soportar los rigores de un nuevo nacimiento y en Bolaño también los sufre pero de un modo distinto. Bolaño decide transitar otro camino e implementa el decir con un gesto que parece ser contrario a lo que hemos visto en Heidegger: su lenguaje se torna sencillo, cotidiano, indiferente, distante, casi vulgar y anodino en ciertos pasajes. En 2666, describiendo la obra del desaparecido escritor alemán Beno Archimboldi -uno de los muchos protagonistas del libro- da cuenta Bolaño de su propio gesto lingüístico de la siguiente manera:

El estilo era extraño, la escritura era clara y en ocasiones incluso transparente pero la manera en que se sucedían las historias no llevaba a ninguna parte: solo quedaban los niños, sus padres, los animales, algunos vecinos y al final, en realidad, lo único que quedaba era la naturaleza, una naturaleza que poco a poco se iba deshaciendo en un caldero hirviendo hasta desaparecer del todo (Bolaño, 2008, p. 1111).

Hay dos elementos clave en este pasaje sobre el carácter del giro lingüístico en Bolaño. El primer elemento guarda relación con el gesto mismo y es expresado de modo doble: por una parte se quiere hacer patente que la claridad y la simpleza que caracterizan el "estilo" de Archimboldi (y del propio Bolaño) no implican que la palabra deba tornarse un instrumento de lo obvio. La sencillez del decir literario puede contener -como se describe en el mismo pasaje- también lo más extraño. El carácter de claro y simple revela además que el lenguaje actúa sin urgencia, pues su objeto no está considerado en riesgo de pérdida irremediable como en Heidegger. La claridad y sencillez delatan cierta ecuanimidad que no necesita recurrir a la elasticidad lingüística para revelar un fondo que es entendido más bien como ineludible. El centro de la obra de Bolaño está oculto, pero no se está escapando, más bien se oculta como lo que acecha.

y finalmente y de modo aún más radical "Seyn" para denotar un regreso al origen del pensamiento ontológico. 


\section{Daniel Michelow Briones}

Por otra parte se expresará la rareza de lo revelado en la concatenación del relato, en "la manera en que se [suceden] las historias". El descubrimiento del centro oculto tiene lugar en una construcción temporal anormal, esto es en la elaboración de un relato no lineal, no anclado al sujeto. Justamente esto último explica la multiplicidad de voces en la obra del chileno: el relato no está atado al destino de un personaje (ni al de muchos), sino que el destino de todos los personajes está atado al centro oculto y solo en la gran variedad de miradas es posible acreditar tal prioridad del centro por sobre el sujeto. En ningún caso se quiere con esto decir que la gran cantidad de voces en el relato de Bolaño tenga como meta dar cuenta de una suprasubjetividad o intersubjetividad, es decir, un mero cambio de un sujeto único (el héroe) a un sujeto múltiple, sino que más bien de una asubjetividad fundamental.

El segundo elemento que se reconoce en este pasaje hace referencia a lo revelado mismo en el relato. Esto es en el contexto de 2666, la naturaleza. Como el propio Heidegger ha tematizado en más de una oportunidad, es la naturaleza, la physis, en su comprensión griega presocrática, el fenómeno fundamental que sustenta el pensar originario. La palabra naturaleza para el entendimiento griego no denota la comprensión actual como el conjunto de las cosas naturales, sino más bien la articulación de los movimientos ontológicos del salir a la vista o brotar (Aufgehen) así como del ocultarse (Untergehen) del ente en su totalidad ${ }^{3}$ (Heidegger, GA 55, 93). Una disposición temporal en que el ente adquiere y pierde todo sentido. Bolaño expresa con el pasaje citado -al modo de la comprensión literaria- que el sentido de una obra poética que reflexiona acerca de sus límites y posibilidades termina siempre y necesariamente relatando no ya los sucesos particulares de los personajes y sus circunstancias (del ente diría Heidegger), sino que fundamentalmente la disposición temporal de ser (el acontecimiento) que permite en primer término la constelación de sucesos, su unidad y fragmentación. Bolaño no pretende describir y disponer hechos dados y significados de antemano, sino que más bien dar cuenta del contexto en el que estos pueden adquirir primeramente todo significado.

Esto nos permite concluir que, por caminos diferentes, tanto Bolaño como Heidegger, sin necesidad de hacerlo expreso y tematizarlo mediante la misma constelación terminológica, comparten, en todo el despliegue de sus obras, la misma comprensión descubridora del lenguaje y el "objeto" de tal función: fundamentalmente hacemos referencia aquí a que el decir, ya sea tanto filosófico como literario, tiene su concreción última en el hacer patente el modo en que el ente está predispuesto para su propio despliegue histórico. Queda por tanto explicar por qué entonces el gesto lingüístico varía tan dramáticamente entre ambos autores.

\footnotetext{
${ }^{3}$ La frase completa a la que hacemos referencia aparece en la traducción de Jaime Echarri (1997): "Solo en la luz del fuego pensado así, en el 'Aufgehen' de la physis entendida así, cada objeto, que aparece, aparece bajo los límites acoplados de su conjunto. El ente en total queda abierto a su 'Aufgehen' por el acoplamiento, es decir queda 'expuesto' 'Aufgemacht' en sentido literal...".
} 


\section{ÁNIMO}

El momento descrito de la filosofia de Heidegger varía significativamente tras lo que él mismo llamará el giro (Kehre) de su pensamiento. Una de las dimensiones más llamativas de esta variación tiene que ver precisamente con el estado de ánimo que impregna su obra. El estado de ánimo de su pensamiento no es ya más el ánimo que acompaña al gesto destructivo, esto es la angustia ${ }^{4}$, sino que en más el de la serenidad (Gelassenheit). Una serenidad respecto de lo que él llamará la época técnica y más precisamente de los objetos técnicos, de poder -como dice el propio Heideggerdecirles que sí y no a la vez (Heidegger, GA 13, 8). El ánimo de su pensar es sereno respecto del sentido técnico que determina lo ente y asimismo el gesto lingüístico. El modo técnico en que todo lo ente está dispuesto y comparece comienza a ser visto en la obra tardía de Heidegger, tras el giro, como un camino en el que necesariamente debemos adentrarnos. La serenidad trata de la necesidad de estar en una predisposición para cierta aceptación, hasta que las condiciones para una modulación diferente del estar revelado del ente, de su Aufgehen, sean posibles.

No se trata ya de imponerse en una "guerra de gigantes" por la verdad, como reza la máxima que da pie a ST (5), sino de dejarla tomar forma en su propio despliegue. Heidegger, al final de su vida, da cuenta de esta transformación del temple de su obra y del lenguaje que lo acompaña. No en el sentido de que cambien las metas propuestas para su proyecto pero sí el enfoque, según asevera él mismo: "Se requiere un nuevo cuidado del lenguaje. No ya la invención de nuevos términos -como yo alguna vez pensé- sino que el regreso al contenido primordial de nuestra propia comprensión de lenguaje, que a la vez se marchita constantemente"5 (Wissel y Rüdel, 1975).

Bajo la pregunta por la unidad de los distintos estados de ánimo que imperan en la obra temprana y la obra tardía de Heidegger se vuelve necesario revelar el momento de articulación de estos. Este momento es en cierto modo la experiencia basal que surge de la más simple y original intuición del ser. Esta experiencia puede ser en Heidegger caracterizada como "añoranza". Tanto la angustia como la serenidad son en este sentido modos de la añoranza. Añoranza de un mundo anterior, de unidad con el ser que parece haber desaparecido bajo un manto de olvido. Si bien tal añoranza no ha sido tematizada por Heidegger, se deja entrever en el recinto conceptual de su comprensión tardía del ser-en-el-mundo con palabras como terruño (Heimat), arraigo (Bodenständigkeit) y apropiación del mundo (Weltaneignung) entre muchos más.

El ser se añora - al modo de la destrucción o de la serenidad- porque se da a entender como el bien perdido. "Bien" no está dicho en un sentido moral, sino que de modo ontológico en su acepción más radical, es decir, como la más originaria relación

\footnotetext{
${ }^{4}$ Utilizamos para determinar el estado de ánimo que caracteriza la obra del primer Heidegger una palabra que él mismo ha recogido. Angst, angustia, no está sin embargo comprendida en su obra de modo particular y autorreflexivo, como se hace aquí, sino que de modo general.

${ }^{5}$ Traducción propia. En el original: "Keine Erfindung neuer Termini, wie ich einmal dachte, sondern ein Rückgang auf den ursprünglichen Gehalt unser eigenen, aber ständig im Absterben begriffenen Sprache".
} 
de pertenencia mutua entre el ser y lo humano. Bien en el sentido en que el mundo presocrático griego decía "armonía".

En términos particulares se puede afirmar entonces que tras la técnica, esa forma histórica del ser mismo que ignora su propio origen, subyace oculta una relación armónica entre el ámbito humano y el ámbito de ser, que de modo distorsionado aún sigue operando. Así la época técnica no puede ser ya más entendida como un peligro insoslayable, sino más bien como un escollo que conlleva su propia forma de esperanza y de resolución. En este sentido no es casualidad que justamente el poema Patmos de Hölderlin sea aquel que ocupa un lugar preponderante en el análisis poético de Heidegger. En este, Hölderlin dice -y Heidegger hace suya- la famosa máxima: "Pero donde está el peligro crece también lo salvador" (Heidegger GA 7, 12). En el peligro que conlleva la técnica de olvidar el ser, crece justamente la condición de posibilidad de preguntar por este nuevamente y además de modos antes nunca intuidos.

Aún así, sufriendo el decir de Heidegger una gran transformación y acercándose al decir literario ecuánime, no se torna en ningún caso "claro y simple" al modo de Bolaño. La serenidad del segundo Heidegger y la ecuanimidad de Bolaño, si bien emparentadas delatan, a la luz de las acentuadas diferencias respecto del gesto, tener su origen en diferentes experiencias basales. En Bolaño, el primer ánimo derivado de tal experiencia es uno radicalmente distinto a la añoranza, que puede ser catalogado incluso como horror. El horror ante la destrucción de lo humano. La ecuanimidad de su lenguaje es -como ya se ha hecho el ejercicio anteriormente respecto de la obra de Heidegger- una modulación de este horror, un modo de ser testigo de lo horroroso sin quedar absorto, sin ser absorbido por él. Se está en esta obra en una comprensión de lo oculto que no requiere un despliegue positivo de búsqueda, sino que más bien su recepción y canalización. La ecuanimidad de la obra de Bolaño es la de lo trágico e ineludible. El mal no se va a buscar sino que más bien se debe soportar. Esto precisamente hace que el gesto de implementación de la función del lenguaje tenga un carácter de clara y simple aceptación.

Bolaño comienza por esta razón su obra principal con la cita de Baudelaire: “¡En desiertos de tedio, un oasis de horror!" (2018, p. 187). Esta frase tiene la clara intención de anunciar el carácter total de la obra que precede. El horror es a su vez la experiencia fundamental que hace Bolaño del centro oculto de los acontecimientos. La experiencia que se hace es de horror ante el mal que acecha lo humano. Son diversas las referencias a este mal en su obra. En una nota, al comienzo de Entre paréntesis afirma: "en Estrella distante, intento una aproximación, muy modesta, al mal absoluto" (Bolaño, 2004, p. 31). Horror y mal son también puestos en una estrecha relación por el propio autor cuando dice que "para salir del aburrimiento, para escapar del punto muerto, lo único que tenemos a mano, y no tan a mano, también en esto hay que esforzarse, es el horror, es decir el mal" (Bolaño, 2003, p. 151).

Horror y mal van de la mano, pues en términos estructurales el primero es el estado de ánimo que produce la más originaria experiencia del centro mismo, del ser. El propio 
autor explica en múltiples ocasiones aquello que se mienta con el término mal al afirmar que "el mal es básicamente el egoísmo narrado de diferentes formas. La frontera la delimita la mirada que tengas sobre el otro, el saber que el otro existe" (Braithwaite, 2006, p. 81), pero además también "[...] el otro no existe, no piensa, no siente, y hacerle daño. O saber que existe, piensa y siente, y hacerle daño" (Gonzales, 2004, p. 28). Pero este mal va mas allá de una crisis moral de esta o de otra sociedad o cultura, es más bien el horror a la absoluta pérdida de valor de la vida humana, el horror a la desaparición del otro en general, al debilitamiento de la alteridad. Se trata del horror ontológico ante el proceso de fragmentación de lo humano. Esto es de que la experiencia de la comunidad de lo humano que es solo posible a partir de la comprensión de mundo emanada desde la experiencia originaria de ser se retire para dejar solo subjetividades individuales. En ese mundo conformado solo de una subjetividad fragmentada, el egoísmo y la destrucción del otro tienen solo límites de tipo puramente formal ${ }^{6}$.

Es en este nivel, en el ánimo, encontramos finalmente el ámbito desde donde se originan las diferencias lingüísticas entre ambas obras, más parecidas de lo que aparentan en un principio. La diferencia no puede ser explicada ya más en términos estilísticos, sino que es necesario recurrir a un análisis del tipo fenomenológico para dar con el fundamento que requiere las formas del decir. En el fondo encontramos lo mismo, sea llamado ser o centro oculto, experimentado de modos diversos y exigiendo modos particulares de expresión.

\section{CONCLUSIONES}

Son varios los aspectos analizables que se desprenden de la presente argumentación. El primero y más obvio es que es solo posible acceder a la totalidad del fenómeno existenciario del decir en la medida en que se dé cuenta de él de modo triple. La trinidad compuesta por función, gesto y ánimo nos permite acceder a aquello que determina el lenguaje de modo más fundamental y por tanto posibilita poner frente a frente diferentes despliegues lingüísticos sin caer en la vaguedad de la comparación estilística. Así no pueden ser ya más comprendidas las diferencias lingüísticas en obras que comparten la función descubridora del lenguaje desde una óptica del tipo genético, que apunta a la generación y desarrollo de las tesis que conducen a un pensamiento y al contexto histórico-filosófico en el que las obras tienen lugar, sino que solo y únicamente de modo fenomenológico, esto es, en este contexto, desde la dación y experiencia de aquello mismo que se pretende descubrir.

En términos específicos lo que queda acreditado mediante las similitudes y diferencias observadas entre Bolaño y Heidegger es que la función reveladora del

\footnotetext{
${ }^{6}$ En el sentido de un mal extramoral es interesante hacer notar el paralelismo con el autor francés Bernard Sichère que expresa de otro modo esta misma idea acerca de un mal que va más allá de la distorsión de valores morales, sino que atañe a la esencia misma del hombre: "[...] el mal, como mal radical, surge paradójicamente como la evidencia de algo extraño y amenazador que el sujeto experimenta y que desde el interior lo quebranta hasta el punto de arrancarlo de su propia cohesión" (Sichère, 1996, p. 19).
} 
lenguaje, que ambas obras comparten, es un momento fundamental de la estructura del decir existenciario, pero que no puede de modo aislado dar cuenta de la totalidad del despliegue del fenómeno lingüístico. La función descubridora no es el indicador central para explicar las divergencias descritas en el gesto. El indicador es más bien el ánimo y la constelación de fenómenos que de él se desprenden. Así se nos presentan en las obras que se han elegido, dos ánimos a analizar: en el caso de Heidegger, la angustia (y más tarde la serenidad) que vienen a expresar la experiencia basal conformada por añoranza y bien ontológico y por otra parte en el caso de Bolaño se trata de la ecuanimidad que se desprende de la experiencia basal horror y mal ontológico. Aquí precisamente radica el origen de las diferencias de sus lenguajes y por tanto también el fundamento para desplegar la pregunta por modos venideros de escritura, esto es por futuros esfuerzos filosóficos y literarios. El modo concreto que adopta el decir no es por tanto nunca una opción superficial, sino que más bien viene mandatado por el modo en que nos corresponde experimentar el ser en su despliegue histórico en el sentido más primario. Esto es como lo que nos salva, como lo que nos destruye, se nos oculta, se vuelve ineludible o está a punto de ser olvidado para siempre.

\section{OBRAS CITADAS}

Adomo, Theodor W. (2005). Jerga de la autenticidad. En: Obras completas 6. Madrid: Akal. Baudelaire, Charles (2018). Las flores del mal. Trad. E.M.S. Danero. Disponible en: http://biblioteca.org.ar/libros/133456.pdf

Bolaño, Roberto (2013). El secreto del mal. Barcelona: Anagrama.

_ (2009). Una novelita lumpen. Barcelona: Anagrama.

— (2008). 2666. Barcelona: Anagrama.

— (2004). Entre paréntesis. Barcelona: Anagrama.

— (2003). "Literatura+Enfermedad: enfermedad", en El Gaucho insufrible. Barcelona: Anagrama.

Braithwaite, Andrés (2006). Bolaño por sí mismo: entrevistas escogidas. Santiago: Diego Portales.

Echarri, Jaime (1997). Fenómeno y verdad en Heidegger. Bilbao: Universidad de Deusto. Echevarría, Ignacio (2004). Nota a la primera edición en: 2666. Barcelona: Anagrama.

Heidegger, Martin (2012). Heráclito. Buenos Aires: El hilo de Ariadna editorial.

— (2002). Serenidad. Barcelona: El Serbal.

_ (2000). Carta sobre el humanismo. Madrid: Alianza.

(1997). Ser y tiempo. Santiago de Chile: Editorial Universitaria.

(1994). Conferencias y artículos. Barcelona: Serbal.

(1992). Hölderlin y la esencia de la poesía. Buenos Aires.

Sichère, Bernard (1996). Historias del mal. Barcelona: Gedisa Editorial.

Wissel, R. \& Rüdel, W. (1975). Directores. Martin Heidegger: Im Denken unterwegs. Wissel prod. 